\title{
Renormalization of bilinear quark operators for the chirally improved lattice Dirac operator
}

\author{
Christof Gattringer ${ }^{1}$, M. Göckeler ${ }^{2,1}$, Philipp Huber ${ }^{3}$ \\ and C. B. Lang ${ }^{3}$ \\ ${ }^{1}$ Institut für Theoretische Physik, \\ Universität Regensburg, D-93040 Regensburg, Germany \\ ${ }^{2}$ Institut für Theoretische Physik, \\ Universität Leipzig, D-04109 Leipzig, Germany \\ ${ }^{3}$ Institut für Theoretische Physik, \\ Universität Graz, A-8010 Graz, Austria
}

August 15, 2021

\begin{abstract}
We compute non-perturbative renormalization constants of fermionic bilinears for the chirally improved lattice fermions in the quenched approximation of QCD. We address finite size effects and the influence of Gribov copies. Our results are presented in the RI' and $\overline{\mathrm{MS}}$ schemes as well as in RGI form and we discuss relations between the renormalization constants implied by chiral symmetry.

After publication we corrected the numerator of the first coefficient of $\alpha_{s}^{3}$ in (24) from 3696847 to 3890527 , which yields a $0.2 \%$ higher value of the conversion coefficient at $\mu=2 \mathrm{GeV}$.
\end{abstract}

PACS: 11.15.Ha, 12.38.Gc

Key words: Lattice field theory, renormalization, chiral symmetry 


\section{Motivation}

Lattice Dirac operators obeying the Ginsparg-Wilson constraint [1] (GWC) provide a lattice formulation of chiral symmetry closest to the continuum form; its violation is local and $\mathcal{O}(a)$. Exact $\mathrm{GW}$-fermion fields are protected by their chiral symmetry [2] and renormalization of operators constructed from them is simpler than for e.g. Wilson fermions. Chiral symmetry implies several relations between renormalization constants. Checking these relations provides an important check of how well chiral symmetry is implemented in a particular lattice formulation of QCD [3].

To be more explicit, let us consider the local, flavor non-singlet quark field bilinear operator

$$
O_{\Gamma} \equiv \bar{u} \Gamma d .
$$

Here $\Gamma$ denotes a Clifford algebra matrix. According to their Lorentz symmetry we denote the five types of $\Gamma$ by $\mathrm{S}, \mathrm{V}, \mathrm{A}, \mathrm{T}$ and $\mathrm{P}$ corresponding to scalar, vector, axial vector, tensor and pseudoscalar $\left(\mathrm{S} \sim \mathbf{1}, \mathrm{V} \sim \gamma_{\mu}, \mathrm{A} \sim \gamma_{\mu} \gamma_{5}\right.$, $\left.\mathrm{T} \sim \frac{i}{2}\left[\gamma_{\mu}, \gamma_{\nu}\right], \mathrm{P} \sim \gamma_{5}\right)$. Chiral symmetry implies in particular $Z_{S}=Z_{P}$ and $Z_{V}=Z_{A}$. For conserved covariant currents, Ward identities give $Z_{V}=1$.

So far only the overlap action [4, 5] provides exact GW-fermions. There are, however, several lattice Dirac operators obeying the GWC approximately (such as fixed point [6] and chirally improved (CI) fermions [7]) or in some limit [8, 9]. Although technically more demanding than standard lattice fermion formulations they are still substantially less expensive (in terms of computer resources) than exact overlap fermions. The Bern-GrazRegensburg (BGR) collaboration [10] has been studying the low-lying hadron spectrum and other properties for fixed point [6] and CI fermions [7, 11].

Results involving renormalized quantities like the pion decay constant, the chiral condensate or the quark masses, need renormalization constants in order to be connected to experiment, usually given in the framework of the $\overline{\mathrm{MS}}$ (modified Minimal Subtraction) renormalization scheme. Here we present and discuss results for the renormalization factors of bilinear operators of type (11) for the CI Dirac operator. A subset of our results, but without discussion of the full calculation, has been used earlier [12].

The chirally improved Dirac operator has been introduced in [7, 11, 13] as an approximate solution of the Ginsparg-Wilson constraint. It is formulated as a truncated series of interaction terms with coefficients depending on the gauge coupling. Within the BGR collaboration it has been used to obtain 
the ground state hadrons in the quenched approximation [10] down to pion masses of $270 \mathrm{MeV}$, and it has been demonstrated to have good chiral properties [14]. The renormalization constants for quark bilinears determined in this work permit a more detailed analysis of the chiral properties of the CI operator.

In Sect. 2 we briefly discuss the method we use for calculating the renormalization constants [15] and its implementation. We then summarize the technical details such as the parameters of the simulations, the chiral limit, volume dependence and gauge fixing ambiguities in Sect. 3, before we present the lattice results in Sect. 4. In order to compare with continuum notations like the $\overline{\mathrm{MS}}$ scheme and to discuss the renormalization scale dependence, we recapitulate the conversion to other renormalization schemes in Sect. 5 and then discuss our results for the converted renormalization constants. We conclude and summarize in Sect. 6.

\section{Method}

We want to compute renormalization constants non-perturbatively on the lattice. For this purpose we need a renormalization scheme which can be implemented in lattice Monte Carlo simulations as well as in continuum perturbation theory. The latter property is necessary to enable the conversion of the lattice results to a more conventional scheme such as $\overline{\mathrm{MS}}$. A renormalization scheme satisfying these requirements is the so-called RI (regularization independent) scheme suggested by Martinelli et al. [15].

In this scheme one studies expectation values of the bilinear quark operators between quark fields at a specific momentum value $p^{2}=\mu^{2}$,

$$
\left.\left\langle p\left|O_{\Gamma}\right| p\right\rangle\right|_{p^{2}=\mu^{2}}
$$

and matches them to the corresponding tree-level matrix element $\left\langle p\left|O_{\Gamma}\right|\right.$ $p\rangle_{0}$. This procedure is expected to work in a window

$$
\Lambda_{\mathrm{QCD}}^{2} \ll \mu^{2} \ll 1 / a^{2}
$$

where discretization effects can be neglected, because the renormalization scale $\mu$ is small compared with the lattice cut-off $1 / a$, and (few-loop) continuum perturbation theory can be used to connect different schemes, because $\mu$ is much larger than the QCD scale parameter $\Lambda_{\mathrm{QCD}}$. For comparing with 
the $\overline{\mathrm{MS}}$ scheme a typical value is $\mu=2 \mathrm{GeV}$. In most calculations one has $a \mu \approx 1$ (or even somewhat larger) and the upper limit is not strictly obeyed. On the other hand, the limit also depends on the scaling properties of the actions involved.

Since (2) is gauge-variant, one has to work in a fixed gauge and must compare the gauge dependent lattice matrix elements with the continuum results in the same gauge. Landau gauge fixing is a suitable choice, but one has to keep in mind that the Gribov copies uncertainty could spoil the comparison. In the lattice calculations one finds little, if any signal of such an effect (see our discussion below in Sect. 3 as well as [16, 17, 18]).

Let us briefly summarize the method following [15] in the modification of [19]. When multiplying (2) with $\left\langle p\left|O_{\Gamma}\right| p\right\rangle_{0}^{-1}$ and taking the trace (note that the object in Eq. (2) is a matrix in color and Dirac space) one obtains for the renormalization condition

$$
\left.Z_{\Gamma} \frac{1}{12} \operatorname{tr}\left[\left\langle p\left|O_{\Gamma}\right| p\right\rangle\left\langle p\left|O_{\Gamma}\right| p\right\rangle_{0}^{-1}\right]\right|_{p^{2}=\mu^{2}}=1
$$

The matrix element

$$
\left\langle p\left|O_{\Gamma}\right| p\right\rangle=\frac{1}{Z_{q}} \Lambda_{\Gamma}(p)
$$

is proportional to the amputated Green function

$$
\Lambda_{\Gamma}(p)=S^{-1}(p) G_{\Gamma}(p) S^{-1}(p),
$$

and $Z_{q}$ is the quark field renormalization constant to be discussed below. The Green function $G_{\Gamma}(p)$ is determined as the expectation value

$$
G_{\Gamma}(p)_{\alpha \beta}=\frac{1}{V} \sum_{x, y} e^{-i p(x-y)}\left\langle u_{\alpha}(x) \sum_{z} O_{\Gamma}(z) \bar{d}_{\beta}(y)\right\rangle .
$$

The indices $\alpha, \beta$ run over color and Dirac indices and $V$ denotes the lattice volume. The quark propagator is

$$
S_{\alpha \beta}(x, y)=\left\langle u_{\alpha}(x) \bar{u}_{\beta}(y)\right\rangle=\left\langle d_{\alpha}(x) \bar{d}_{\beta}(y)\right\rangle
$$

(assuming that $u$ and $d$ have equal masses and using the Landau gauge for the expectation value).

So we have to compute $G_{\Gamma}(p)$ and $S(p)$. This is done in the following way. For the quark propagator $S_{n}$ evaluated on a single gauge configuration $n$ we define

$$
S_{n}(x \mid p)=\sum_{y} e^{i p y} S_{n}(x, y)
$$


Taking into account $\gamma_{5}$-hermiticity of the propagator we may, for quark bilinear operators $O_{\Gamma}$ as defined in Eq. (1) , rewrite $G_{\Gamma}(p)$ in terms of the quantities (9),

$$
\begin{aligned}
G_{\Gamma}(p) & =\frac{1}{V} \sum_{x, y, z} e^{-i p(x-y)}\langle u(x) \bar{u}(z) \Gamma d(z) \bar{d}(y)\rangle \\
& \approx \frac{1}{V N} \sum_{n=1}^{N} \sum_{z} \gamma_{5} S_{n}(z \mid p)^{\dagger} \gamma_{5} \Gamma S_{n}(z \mid p)
\end{aligned}
$$

averaging over $N$ gauge configurations. Similarly we find the quark propagator in momentum space

$$
S(p) \approx \frac{1}{V N} \sum_{n=1}^{N} \sum_{x} e^{-i p x} S_{n}(x \mid p) .
$$

$S_{n}(y \mid p)$ is computed by solving the lattice Dirac equation $\left(D_{C I}\right.$ denotes the chirally improved Dirac operator)

$$
\sum_{y} D_{C I}(z, y) S_{n}(y \mid p)=e^{i p z}
$$

with a momentum source (cf. [19]). This has the disadvantage that one has to determine the quark propagators for several momentum sources, whereas in the original method [15] one uses point sources (i.e. taking into account just $z=0$ instead of summing over all $z$ ) and projects the quark sink to the desired momentum values. However, using momentum sources has the big advantage of a significantly better signal.

The quark field renormalization constant is obtained by comparing the quark propagator to the free (lattice) propagator. Using the so-called RI' scheme we take

$$
Z_{q}^{\prime}=\left.\frac{1}{12} \operatorname{tr}\left(S^{-1}(p) \frac{\mathbf{1} R(p)-i \gamma_{\mu} a_{\mu}(p)}{R(p)^{2}+\sum_{\mu} a_{\mu}(p)^{2}}\right)\right|_{p^{2}=\mu^{2}}
$$

Here $R$ and $a_{\mu}$ are the scalar and vector terms appearing in the free CI Dirac operator, which in momentum space reads $D_{C I}(p)=i \gamma_{\mu} a_{\mu}(p)+R(p)$. Using Fourier transformation one can compute $R$ and $a_{\mu}$ from the definition and the parameters in $D_{C I}$ [7, 13]. They are normalized such that one finds

$$
a_{\mu}(p)=i p_{\mu}+\mathcal{O}(a p)^{2} \text { and } R(p)=\mathcal{O}(a p)^{2}
$$


Landau gauge fixing $\partial_{\mu} A_{\mu}=0$ is implemented as discussed in [20, 17] by iteratively minimizing a functional of the link variables with stochastic overrelaxation [21]. As is well known this type of gauge fixing still allows for Gribov copies. This gives rise to an uncertainty which we address in Sect. 3.3 .

It is easy to check that at tree level one finds $\left\langle p\left|O_{\Gamma}\right| p\right\rangle_{0}=\Gamma$. Putting things together we obtain the final formula for $Z_{\Gamma}$ in the RI' scheme

$$
Z_{\Gamma}^{\mathrm{RI}}=\left.\frac{12 Z_{q}^{\prime}}{\operatorname{tr}\left[\Lambda_{\Gamma}(p) \Gamma^{-1}\right]}\right|_{p^{2}=\mu^{2}} .
$$

For $\Gamma=\gamma_{\nu}, \gamma_{\nu} \gamma_{5}$ averaging over the index $\nu$ under the trace is implied. The relation between different schemes is discussed in Sect. 5 .

\section{Technicalities}

\subsection{Parameters}

The gauge configurations were generated in the quenched approximation with the Lüscher-Weisz action 22] at values of the gauge coupling $\beta=7.90,8.35$ and 8.70 corresponding to lattice spacings of $a=0.148 \mathrm{fm}=0.750 \mathrm{GeV}^{-1}$, $a=0.102 \mathrm{fm}=0.517 \mathrm{GeV}^{-1}$ and $a=0.078 \mathrm{fm}=0.395 \mathrm{GeV}^{-1}$, respectively [23]. The scale has been determined from the Sommer parameter $r_{0}$.

The final numbers we quote were computed on lattices of size $16^{3} \times 32$. For studying possible volume and gauge fixing dependence we used additional ensembles on $8^{3} \times 24$ at $\beta=7.90$. More details on the simulations can be found in [10].

For each lattice size and gauge coupling we determined the quark propagators $S(x \mid p)$ on different (gauge fixed) gauge configurations and for typically 16 different momentum sources. We used 11 different values for the quark masses, ranging from $a m=0.01$ up to 0.20 covering a range of pion masses from $250 \mathrm{MeV}$ up to $1-2 \mathrm{GeV}$ (depending on $\beta$ ). The boundary conditions for the fermions were chosen periodic in space and anti-periodic in the time direction. The momentum values were taken to lie roughly along the diagonal of the Brillouin zone ranging from the origin up to $p=(5,5,6,10) 2 \pi / L$ with values of $a \mu$ up to 4.18 .

For each mass and each operator $O_{\Gamma}$ we evaluated $Z_{\Gamma}^{\mathrm{RI}}$ from (16). On the small lattice we used 10 gauge configurations and on the larger ones we 
had 5. We calculated the statistical error with the help of the statistical bootstrap method.

\subsection{Extrapolation to the chiral limit}

As we want to obtain a mass-independent scheme we extrapolate all results for $Z_{\Gamma}^{\mathrm{RI}}\left(\mu^{2}, m\right)$ linearly to the chiral limit $m \rightarrow 0$. In Fig. 1 this extrapolation is shown for three representative values of $\mu^{2}$. Whereas the linear fit is appropriate for most cases, we do find a substantially different behavior for the pseudoscalar density. Similar observations have been discussed in [19, 24, 25, 26].

Since the pseudoscalar density couples to the Goldstone boson channel there are $\mathcal{O}(1 / m)$ contributions to $G_{P}$ in the chiral limit. We can incorporate this fact by expanding the inverse renormalization constant [24] in terms of the mass and adding the pole term; in [24] only a pole term and a constant term were used, but one can also add terms $\mathcal{O}(m)$ and higher, which vanish in the chiral limit (cf. [26]). We use

$$
\frac{1}{Z_{P}\left(\mu^{2}, m\right)} \approx \frac{A\left(\mu^{2}\right)}{m}+B\left(\mu^{2}\right)+C\left(\mu^{2}\right) m .
$$

By subtracting the pole term first and then performing the chiral limit we can define a "subtracted" renormalization constant

$$
\frac{1}{Z_{P}^{\operatorname{Sub}}\left(\mu^{2}\right)}=\lim _{m \rightarrow 0}\left(\frac{1}{Z_{P}\left(\mu^{2}\right)}-\frac{A\left(\mu^{2}\right)}{m}\right)=\lim _{m \rightarrow 0}\left(B\left(\mu^{2}\right)+C\left(\mu^{2}\right) m\right)=B\left(\mu^{2}\right),
$$

such that the desired value is

$$
Z_{P}^{\mathrm{Sub}}\left(\mu^{2}\right)=\frac{1}{B\left(\mu^{2}\right)}
$$

The fit to $Z_{P}$ on the large lattices, as shown in Fig. 1, is very satisfactory. For the small lattice, which we use only to study the finite size dependence, the fit is not as good for the smaller values of $\mu^{2}$ (cf. Subsection 3.4).

For large $\mu$ the operator product expansion guarantees the suppression of the pole contributions [15]. This behavior is clearly exhibited in the plot for $Z_{P}$ when comparing the results for smaller $\mu^{2}$ to larger values of $\mu^{2}$. In terms of the expansion coefficients this means that $A\left(\mu^{2}\right)$ approaches 0 fast enough for higher $\mu^{2}$. The appropriate order of limits would be first $\mu \rightarrow \infty$ 


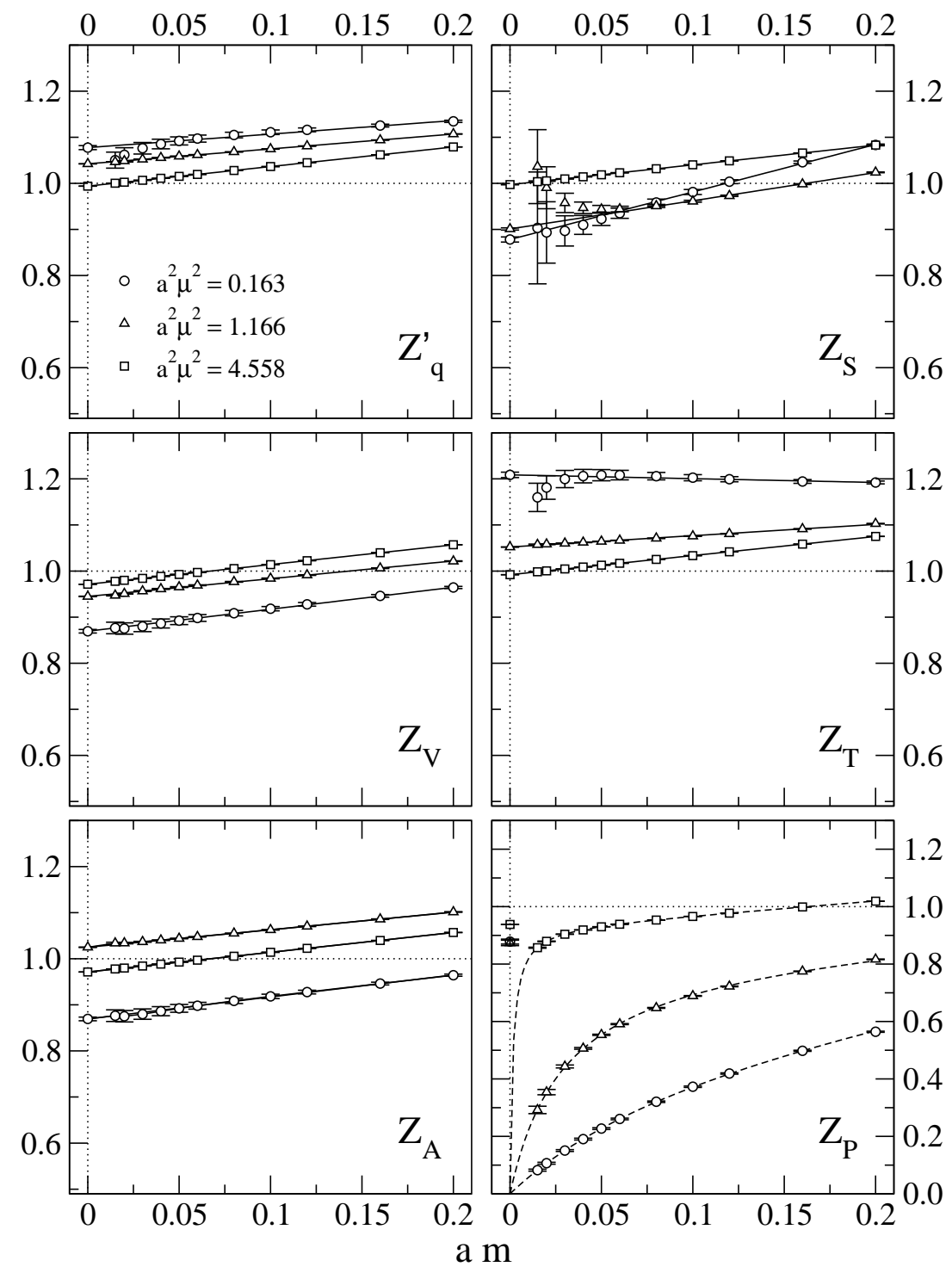

Figure 1: Results for $\beta=7.90$ and volume $16^{3} \times 32$ : The values for $Z_{q}^{\prime}$ (the fermion field renormalization constant), $Z_{S}, Z_{V}, Z_{T}, Z_{A}, Z_{P}$ are plotted versus the quark mass at $a^{2} \mu^{2}=0.163$ (circles), 1.166 (triangles), 4.558 (squares) together with extrapolations to the chiral limit. For $Z_{P}$ the nonlinear fit function (17) is included (dashed line). The points at $m=0$ resulting from the corresponding subtracted extrapolation $Z_{P}^{\mathrm{Sub}}\left(\mu^{2}\right)$ are also shown; the two values for the lower $a^{2} \mu^{2}$ overlap. 
$(a \rightarrow 0)$, then $m \rightarrow 0$. Here, due to the requirements of a lattice calculation, we first perform $m \rightarrow 0$.

The axial vector also couples to the Goldstone boson. However, the coupling is proportional to the momentum transfer [27], which vanishes for our kinematics.

For the further analysis we use the values in the chiral limit.

\subsection{Gauge fixing dependence}

Let us now analyze the effects due to gauge fixing ambiguities. As discussed above we fix our gauge configurations to Landau gauge using overrelaxation. If we apply a random gauge transformation to the original gauge configuration and then fix the gauge again, the procedure often leads to a different (gauge-equivalent) configuration. When comparing the results for the renormalization constants from the two gauge copies we find small differences. This non-uniqueness is the Gribov uncertainty. We study the fluctuations induced by Gribov copies by comparing results for $Z_{\Gamma}^{\mathrm{RI}}\left(\mu^{2}, m\right)$ derived with and without additional random gauge transformations before applying the gauge fixing procedure. In Fig. 2 we plot the ratio

$$
Q_{\Gamma}=\frac{Z_{\Gamma}^{1}-Z_{\Gamma}^{2}}{Z_{\Gamma}^{1}+Z_{\Gamma}^{2}},
$$

(where the superscript denotes the results from the two "different" configuration ensembles) as obtained for $8^{3} \times 24$ lattices at $\beta=7.90$ with $Z_{\Gamma}^{i}$ taken in the chiral limit. For the values of $\mu^{2}$ of interest (around $4 \mathrm{GeV}^{2}$ ) we find these effects to be substantially smaller than $1 \%$. This result agrees with earlier studies [16, 18].

\subsection{Finite size effects}

We use two different lattice sizes in order to study the volume dependence of the renormalization constants. In Fig. 3 we compare the values (extrapolated to the chiral limit as discussed above) for the RI' scheme at $\beta=7.90$. The deviations are generally small, increasing towards smaller momenta as expected for finite size effects. However, above $\mu^{2} \approx 4 \mathrm{GeV}^{2}$ the difference is generally less than $1 \%$ except for $Z_{P}^{\mathrm{Sub}}$. A possible explanation of this exceptional behavior might be that the coupling to the light pions enhances 


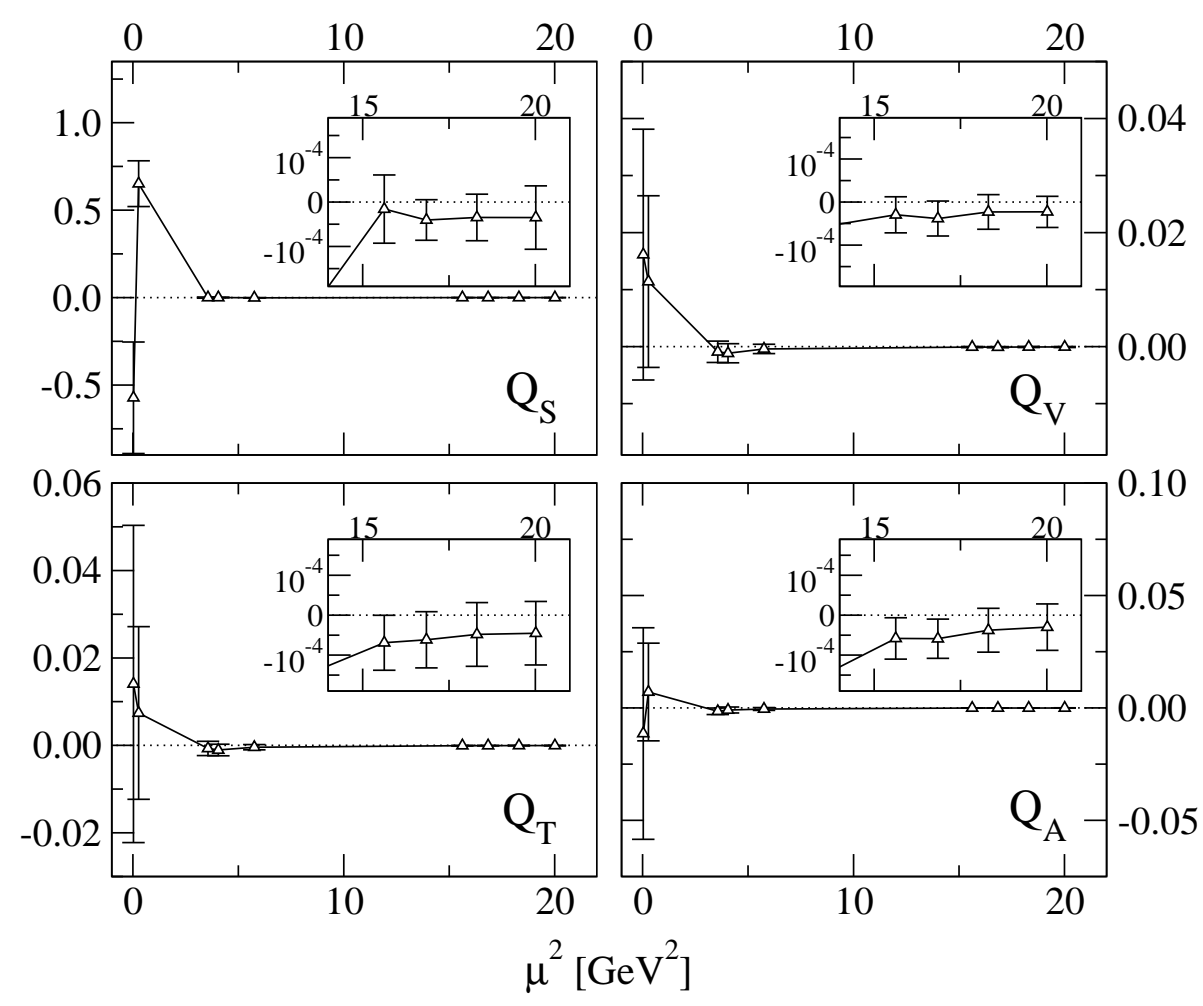

Figure 2: Values for the ratios $Q_{S}, Q_{V}, Q_{T}, Q_{A}$, defined in Eq. (20), vs. $\mu^{2}\left[\mathrm{GeV}^{2}\right]$ for the $8^{3} \times 24$ lattice at $\beta=7.90$.

the finite size effects for $Z_{P}^{\mathrm{Sub}}$. As mentioned in the discussion on the chiral limit, the extrapolation for $Z_{P}$ on the small lattices, in particular for small values of $\mu^{2}$, is not as good as on the larger lattices. This explains the larger error bars for lower $\mu^{2}$ in this case.

We restrict ourselves to the results from the largest lattice of size $16^{3} \times 32$ from now on.

\section{Results for RI'}

In the appendix we collect our results for the chirally extrapolated values of $Z_{\Gamma}^{\mathrm{RI}}$ in tabular form. Figs. 4-6] summarize these data. We discuss in this section only the RI' values (triangles in Figs. 446) ). 


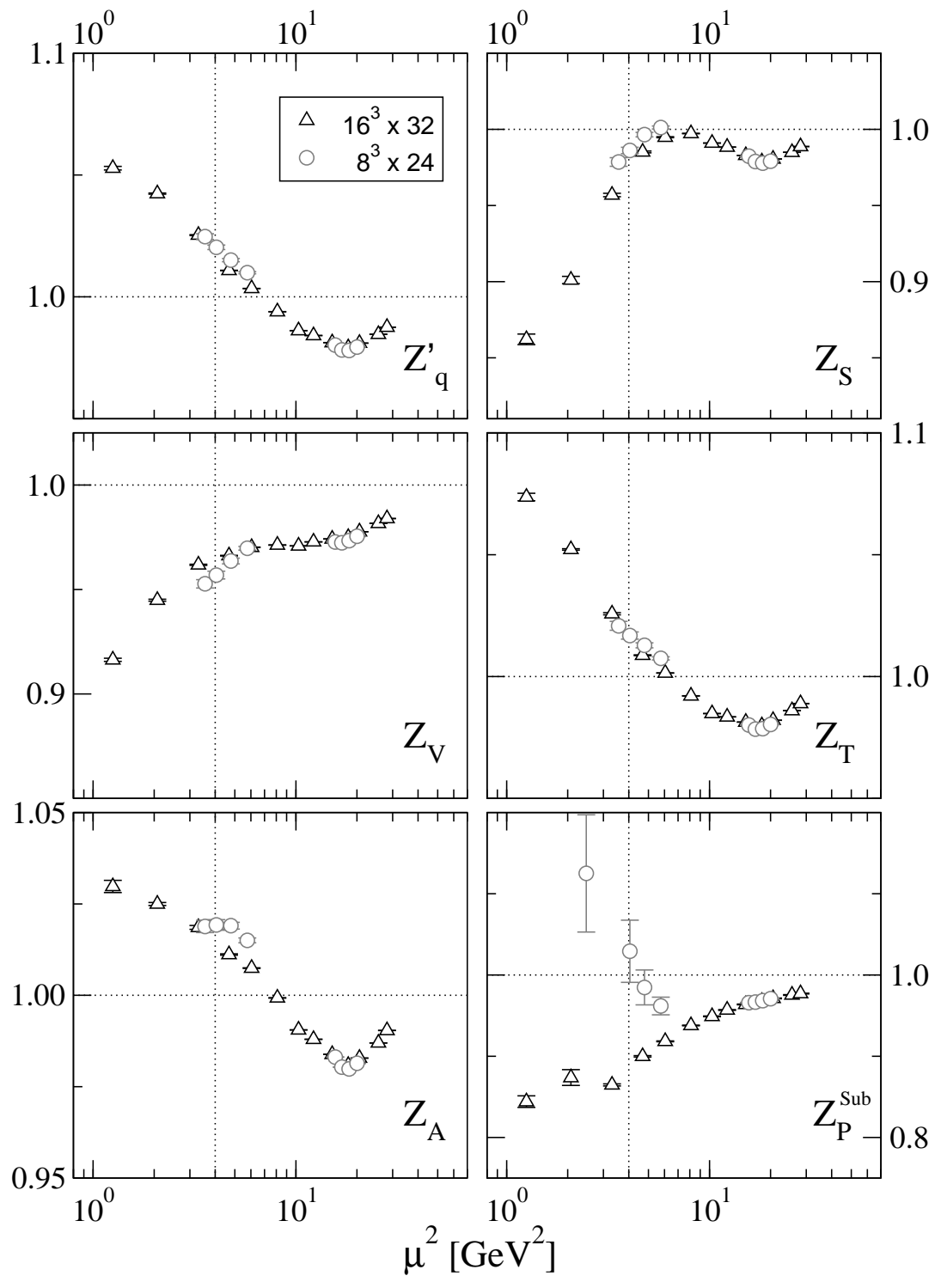

Figure 3: Values for $Z_{q}^{\prime}, Z_{S}, Z_{V}, Z_{T}, Z_{A}, Z_{P}^{\text {Sub }}$ vs. $\mu^{2}$ at $\beta=7.90$. The triangles mark the values for the $16^{3} \times 32$ lattice and the circles the values for the $8^{3} \times 24$ lattice. $Z_{P}^{\text {Sub }}$ is the value determined according to Eq. (19). 


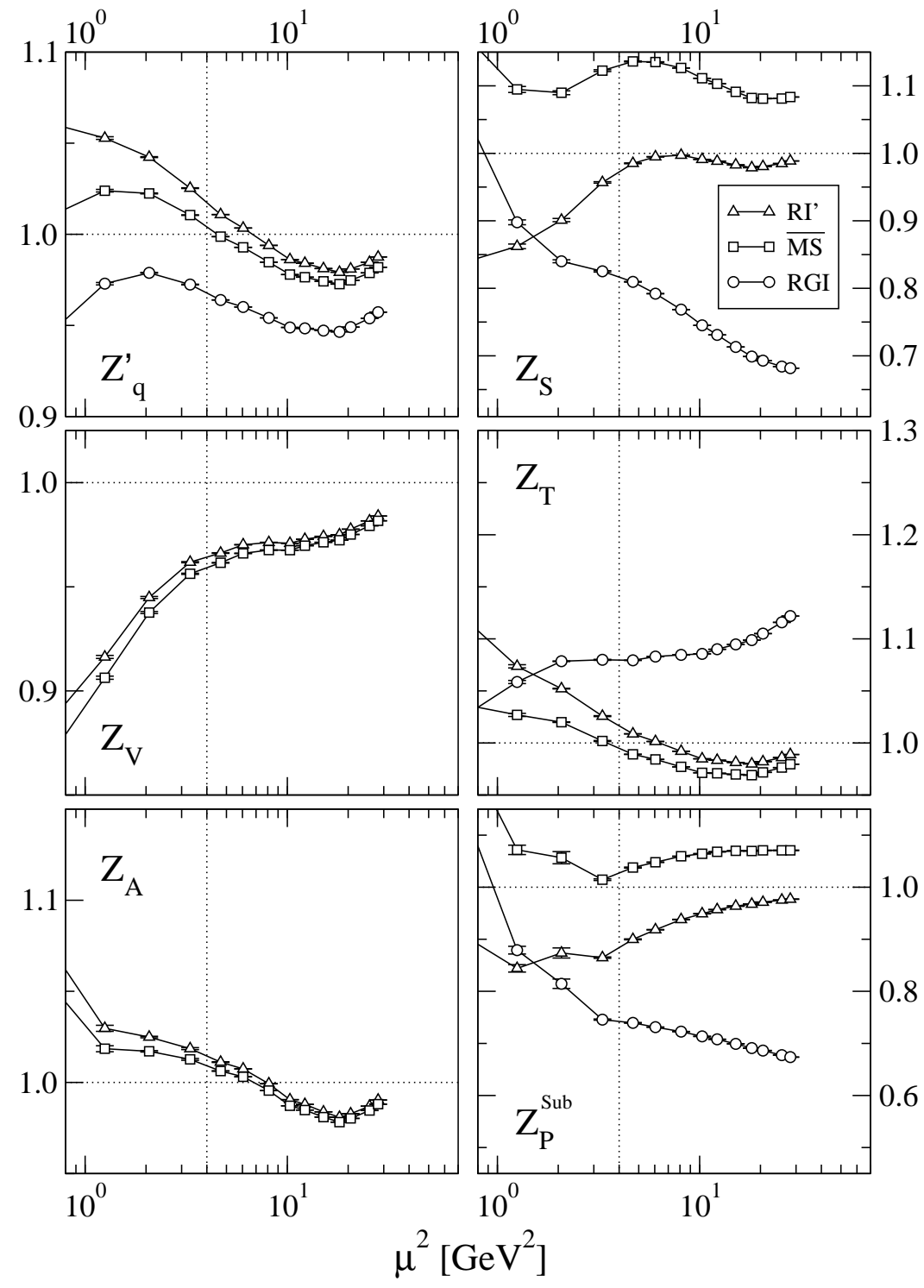

Figure 4: Values for $Z_{q}^{\prime}, Z_{S}, Z_{V}, Z_{T}, Z_{A}, Z_{P}^{\text {Sub }}$ vs. $\mu^{2}\left[\mathrm{GeV}^{2}\right]$ for the $16^{3} \times 32$ lattice at $\beta=7.90$. RI' values (triangles), $\overline{\mathrm{MS}}$ values (squares) and RGI values (circles) are displayed. The dotted vertical line indicates the value $\mu=2 \mathrm{GeV}$ where the $\overline{\mathrm{MS}}$ value has been obtained. Note that for the vector and axial vector the $\overline{\mathrm{MS}}$ and the RGI values coincide. 


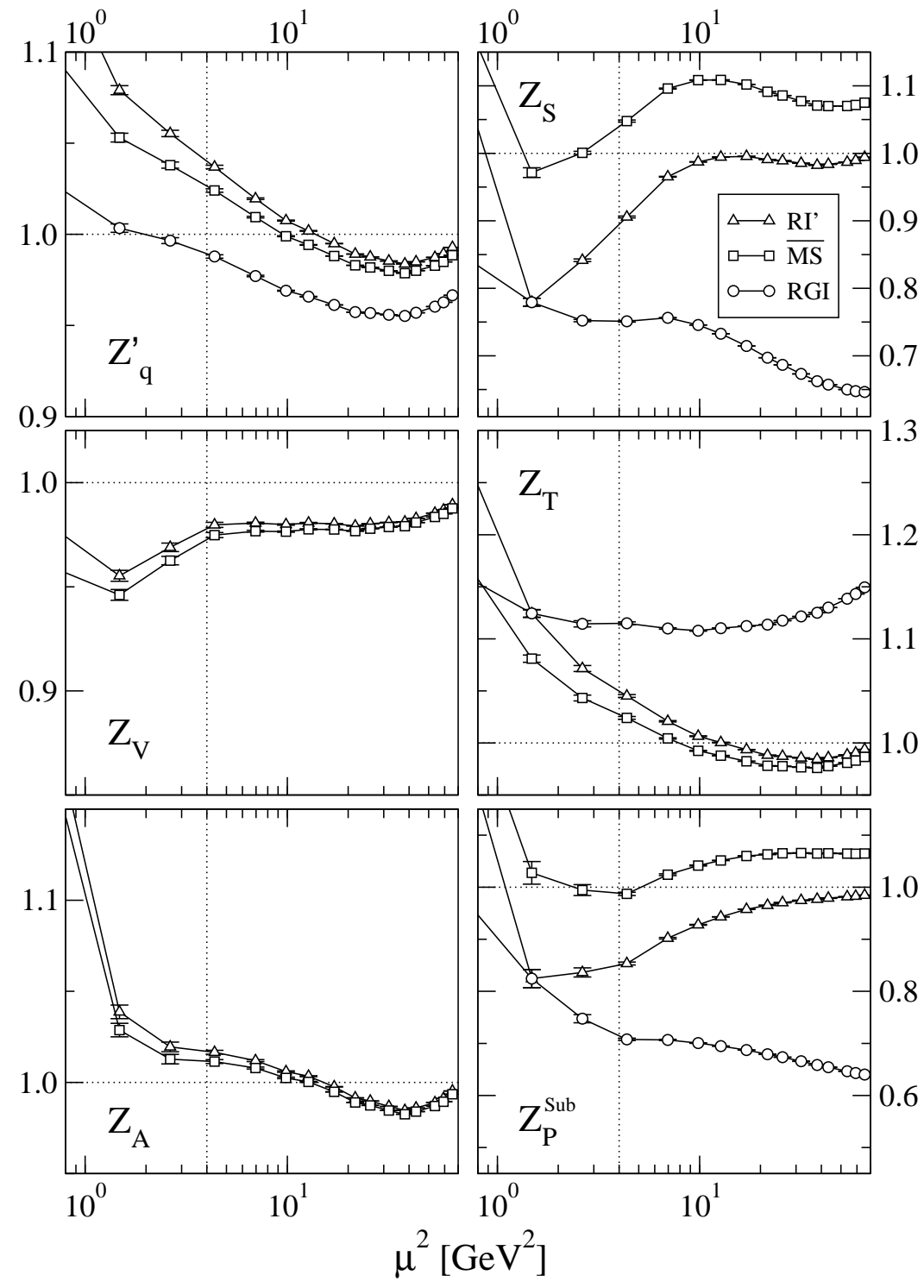

Figure 5: Values for $Z_{q}^{\prime}, Z_{S}, Z_{V}, Z_{T}, Z_{A}, Z_{P}^{\text {Sub }}$ vs. $\mu^{2}\left[\mathrm{GeV}^{2}\right]$ for the $16^{3} \times 32$ lattice at $\beta=8.35$. RI' values (triangles), $\overline{\mathrm{MS}}$ values (squares) and RGI values (circles) are displayed. The dotted vertical line indicates the value $\mu=2 \mathrm{GeV}$ where the $\overline{\mathrm{MS}}$ value has been obtained. Note that for the vector and axial vector the $\overline{\mathrm{MS}}$ and the RGI values coincide. 


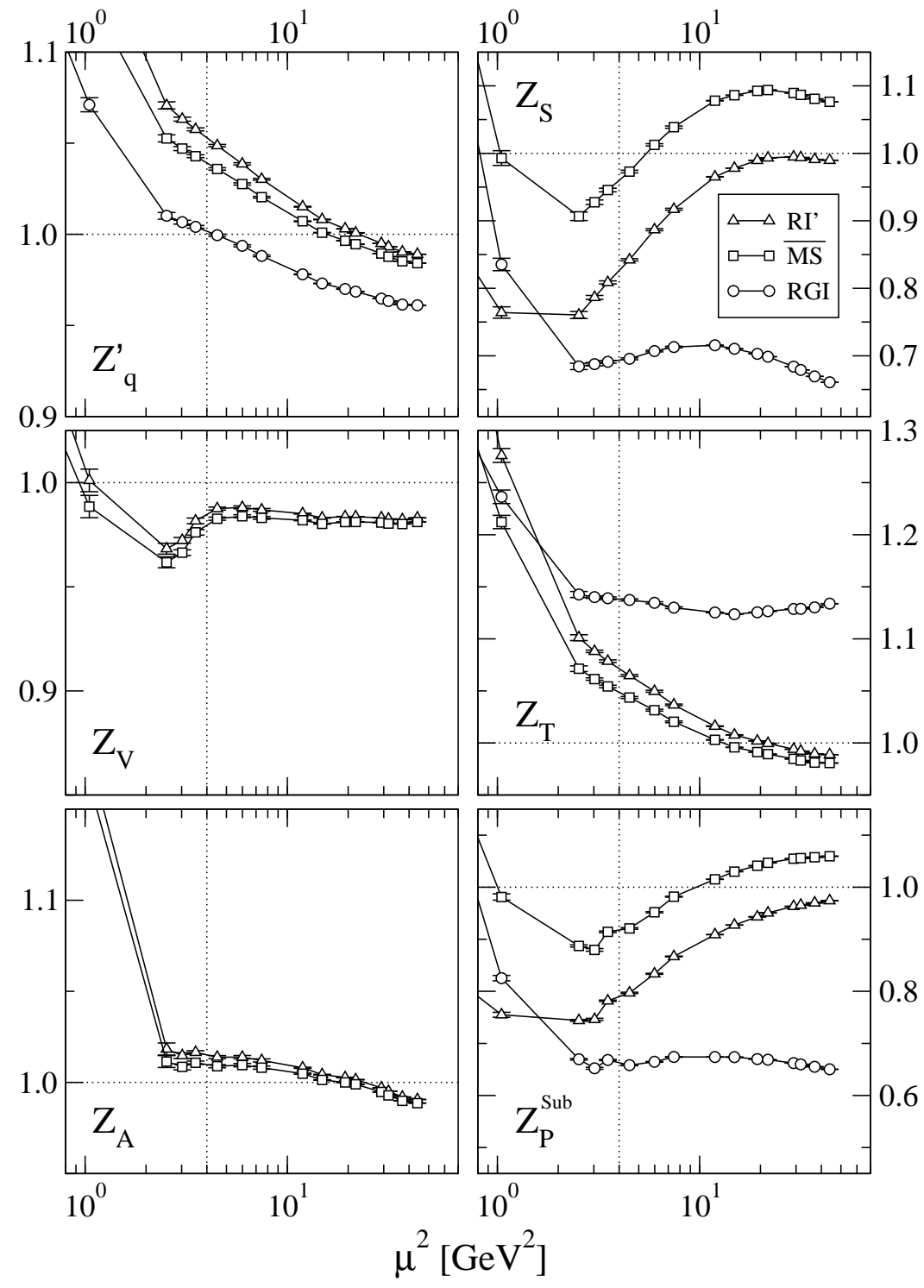

Figure 6: Values for $Z_{q}^{\prime}, Z_{S}, Z_{V}, Z_{T}, Z_{A}, Z_{P}^{\text {Sub }}$ vs. $\mu^{2}\left[\mathrm{GeV}^{2}\right]$ for the $16^{3} \times 32$ lattice at $\beta=8.70$. RI' values (triangles), $\overline{\mathrm{MS}}$ values (squares) and RGI values (circles) are displayed. The dotted vertical line indicates the value $\mu=2 \mathrm{GeV}$ where the $\overline{\mathrm{MS}}$ value has been obtained. Note that for the vector and axial vector the $\overline{\mathrm{MS}}$ and the RGI values coincide. 
Vector and axial vector have finite renormalization factors and should be scale independent. Indeed the changes with $\mu^{2}$ (and also with the lattice size) are distinctly smaller than for the scalar or pseudoscalar renormalization factors. We also compared the variation of the renormalization factor for individual components of $V_{\mu}$ and $A_{\mu}$ and the components of $T_{\mu \nu}$. For $\mu^{2}>$ $4 \mathrm{GeV}^{2}$ the deviation from the mean value is smaller than $1.5 \%$ for all gauge couplings studied, for $\mu^{2}>10 \mathrm{GeV}^{2}$ it is less than $0.3 \%$. We therefore plot and list the average over the results for these components according to the remark following (16).

Our currents are point-like and not the conserved currents. However, the values for the renormalization constants for vector and axial vector are both very close to 1 , a behavior expected for conserved currents.

The scalar and pseudoscalar densities are logarithmically divergent and have scale dependent renormalization. Their ratio, however, should be scale independent. Fig. 7 displays both ratios, $Z_{A} / Z_{V}$ and $Z_{P}^{\mathrm{Sub}} / Z_{S}$. Both ought to be 1 for chirally symmetric fermion actions. Indeed, the observed scale dependence of $Z_{P}^{\text {Sub }}$ and $Z_{S}$ cancels in the ratio. Both ratios are surprisingly close to 1 with very little scale dependence, indicating the excellent approximation to chiral symmetry of the considered CI fermion action.

Only for the two ensembles with smaller lattice spacing $(\beta=8.35, \beta=$ 8.7) we have a reasonable chance to satisfy the limits (3). However, the relatively flat shape of the scale independent $Z_{A}$ and $Z_{V}$ and the analysis of the $\mu$ dependence in the subsequent section indicate that we may still rely on the results around $\mu \approx 2 \mathrm{GeV}$. In fact, due to earlier results [10] we expect that the Dirac operator used here has improved scaling properties even for larger lattice spacings.

\section{Conversion to RGI and $\overline{\mathrm{MS}}$}

\subsection{Different renormalization schemes}

The connection between different renormalization schemes is established using continuum perturbation theory. In the two schemes $\overline{\mathrm{MS}}$ and RI' the renormalization factors are related by

$$
R_{\Gamma}\left(\mu^{2}\right)=\frac{Z_{\Gamma}^{\overline{\mathrm{MS}}}\left(\mu^{2}\right)}{Z_{\Gamma}^{\mathrm{RI}}\left(\mu^{2}\right)} .
$$




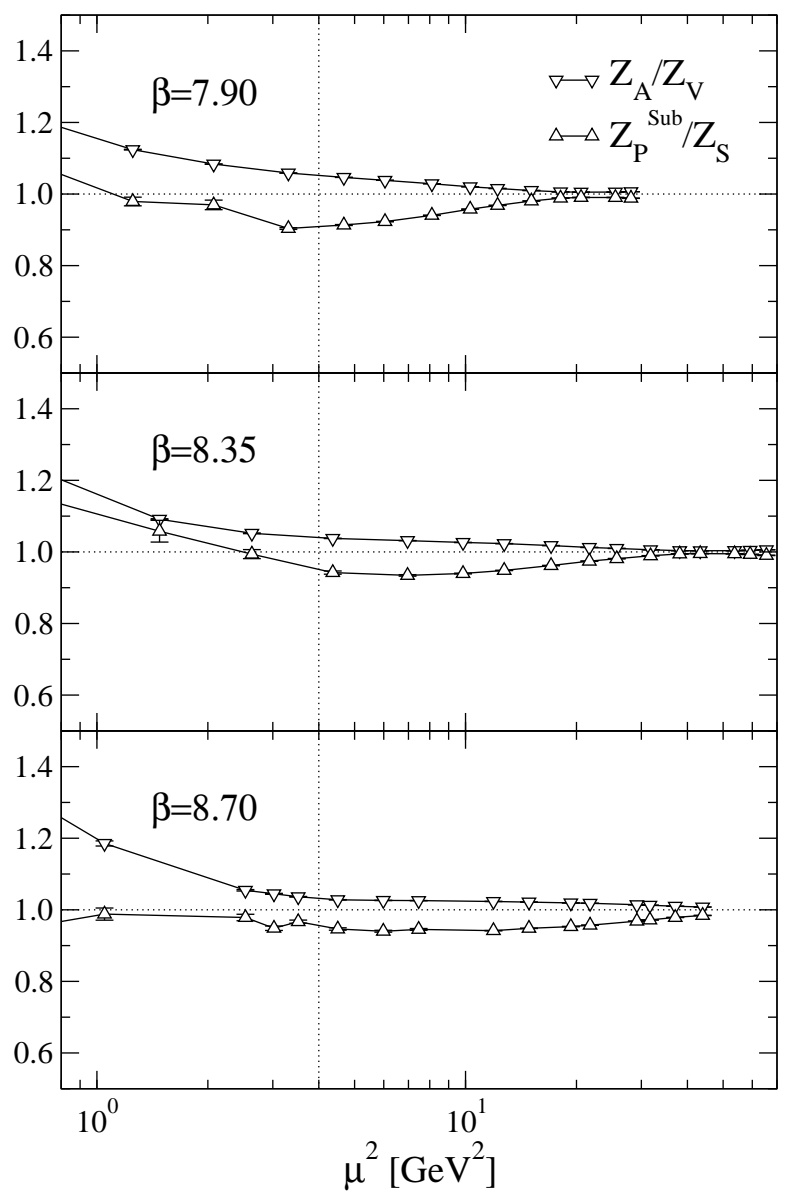

Figure 7: Ratios $Z_{A} / Z_{V}$ and $Z_{P}^{\text {Sub }} / Z_{S}$ for the data in the chiral limit (lattice size $16^{3} \times 32$ ). 
Both, $Z_{\Gamma}^{\mathrm{RI}}\left(\mu^{2}\right)$ and $Z_{\Gamma}^{\overline{\mathrm{MS}}}\left(\mu^{2}\right)$ are in general divergent in the continuum limit, the ratio stays finite, though.

Note that the RI' scheme which we are employing differs from the RI scheme only by the definition of the quark field renormalization constant. The ratio of the latter in the two schemes

$$
\frac{Z_{q}^{\mathrm{RI}}}{Z_{q}^{\prime}}=\frac{Z_{\Gamma}^{\mathrm{RI}}}{Z_{\Gamma}^{\mathrm{RI}}}
$$

agrees with the conversion factor for the vector and axial vector renormalization constants from RI' to $\overline{\mathrm{MS}}$ since there is no further renormalization due to the protection by Ward identities. In the Landau gauge and 3-loop order one has [28, 29]

$$
\frac{Z_{V}^{\mathrm{RI}}}{Z_{V}^{\mathrm{RI}}}=\frac{Z_{A}^{\mathrm{RI}}}{Z_{A}^{\mathrm{RI}}}=1-\frac{67}{6}\left(\frac{\alpha_{s}}{4 \pi}\right)^{2}-\left(\frac{52321}{72}-\frac{607 \zeta_{3}}{4}\right)\left(\frac{\alpha_{s}}{4 \pi}\right)^{3}+O\left(\alpha_{s}^{4}\right) .
$$

Here $\zeta_{n}$ is the Riemann zeta function evaluated at $n$. This equation refers to the average over the different directional components of the currents (under the trace in (16)). For the scalar and pseudoscalar one finds

$$
\begin{aligned}
\frac{Z_{P}^{\overline{\mathrm{MS}}}}{Z_{P}^{\mathrm{RI}}}=\frac{Z_{S}^{\overline{\mathrm{MS}}}}{Z_{S}^{\mathrm{RI}}}= & 1+\frac{16}{3} \frac{\alpha_{s}}{4 \pi}+\left(\frac{4291}{18}-\frac{152 \zeta_{3}}{3}\right)\left(\frac{\alpha_{s}}{4 \pi}\right)^{2} \\
& +\left(\frac{3890527}{324}-\frac{224993 \zeta_{3}}{54}+\frac{2960 \zeta_{5}}{9}\right)\left(\frac{\alpha_{s}}{4 \pi}\right)^{3}+O\left(\alpha_{s}^{4}\right) .
\end{aligned}
$$

In order to illustrate the orders of magnitude involved we give a numerical value at the coupling $\alpha_{s}(\mu=2 \mathrm{GeV})=0.203576$ :

$$
\frac{Z_{S}^{\overline{\mathrm{MS}}}}{Z_{S}^{\mathrm{RI}}} \approx 1.16165
$$

The correction for the different definitions of the quark field renormalization is rather small $(\approx 0.5 \%)$, whereas even the $\alpha_{s}^{3}$ contribution to the scheme conversion is large $(\approx 2.9 \%)$ in comparison.

Concerning the tensors, the conversion factor from RI' to $\overline{\mathrm{MS}}$ has been computed in [30]. In Landau gauge one finds

$$
\begin{aligned}
\frac{Z_{T}^{\overline{\mathrm{MS}}}=}{Z_{T}^{\mathrm{RI}}}= & 1+\left(-\frac{3847}{54}+\frac{184 \zeta_{3}}{9}\right)\left(\frac{\alpha_{s}}{4 \pi}\right)^{2}+ \\
& \left(-\frac{9858659}{2916}+\frac{678473 \zeta_{3}}{486}+\frac{1072 \zeta_{4}}{81}-\frac{10040 \zeta_{5}}{27}\right)\left(\frac{\alpha_{s}}{4 \pi}\right)^{3}+\mathcal{O}\left(\alpha_{s}^{3}\right) .
\end{aligned}
$$


We compute the coupling $\alpha_{s}$ from the 3-loop expression in the $\overline{\mathrm{MS}}$ scheme

$$
\begin{aligned}
\frac{\alpha_{s}\left(q^{2}\right)}{4 \pi}= & \frac{1}{\beta_{0} \log \left(q^{2}\right)}-\frac{\beta_{1}}{\beta_{0}^{3}} \frac{\log \log \left(q^{2}\right)}{\log ^{2}\left(q^{2}\right)} \\
& +\frac{1}{\beta_{0}^{5} \log ^{3}\left(q^{2}\right)}\left(\beta_{1}^{2} \log ^{2} \log \left(q^{2}\right)-\beta_{1}^{2} \log \log \left(q^{2}\right)+\beta_{2}^{\overline{\mathrm{MS}}} \beta_{0}-\beta_{1}^{2}\right),
\end{aligned}
$$

where we have introduced the shorthand notation $q^{2}=\mu^{2} /\left(\Lambda_{\mathrm{QCD}}^{\overline{\mathrm{MS}}}\right)^{2}$. For $\Lambda_{\mathrm{QCD}}^{\overline{\mathrm{MS}}}$ in the quenched approximation we use the value $\Lambda_{\mathrm{QCD}}^{\overline{\mathrm{MS}}}=0.238 \pm$ $0.019 \mathrm{GeV}$ calculated in 31].

In Figs. [4 6 we plot our results converted to the $\overline{\mathrm{MS}}$ scheme. Except for the vector currents we expect dependence on both, $\mu^{2}$ and lattice spacing.

In Tables $1-3$ the $\overline{\mathrm{MS}}$ values are given at $\mu=2 \mathrm{GeV}$ determined by linear interpolation between the closest $\mu$ values of the data.

\subsection{RGI values}

The renormalization factors depend on the renormalization scale $\mu$ (except for the vector and axial currents). This dependence is governed by the anomalous dimension $\gamma$ of the operator:

$$
\gamma\left(\alpha_{s}\right)=-\mu \frac{\mathrm{d}}{\mathrm{d} \mu} \ln Z\left(\mu^{2}\right)=\sum_{i=0}^{\infty} \gamma_{i}\left(\frac{\alpha_{s}\left(\mu^{2}\right)}{4 \pi}\right)^{i+1} .
$$

Integrating this differential equation we can define a scale-independent quantity $Z^{\text {RGI }}$ (RGI = Renormalization Group Invariant) by [32]

$$
Z^{\mathrm{RGI}}=Z\left(\mu^{2}\right)\left(2 \beta_{0} \frac{\alpha_{s}\left(\mu^{2}\right)}{4 \pi}\right)^{-\gamma_{0} /\left(2 \beta_{0}\right)} \exp \left\{\frac{1}{2} \int_{0}^{\alpha_{s}\left(\mu^{2}\right)} \mathrm{d} \alpha\left(\frac{\gamma(\alpha)}{\beta(\alpha)}+\frac{\gamma_{0}}{\beta_{0} \alpha}\right)\right\},
$$

where the $\beta$ function is given by

$$
\beta\left(\alpha_{s}\right)=\frac{\mu}{2} \frac{\mathrm{d}}{\mathrm{d} \mu} \alpha_{s}\left(\mu^{2}\right)=-4 \pi \sum_{i=0}^{\infty} \beta_{i}\left(\frac{\alpha_{s}\left(\mu^{2}\right)}{4 \pi}\right)^{i+2} .
$$

Since we know the renormalization group functions $\beta$ and $\gamma$ only to a certain order in perturbation theory we cannot expect exact scale invariance when we apply the above definitions to our data. However, we can check how well the expectation of $\mu$-independence is met [19]. 
In Figs. 4,6 we also show the RGI values as obtained by converting at each value of $\mu^{2}$ (indicated by circles). For perfect 3-loop scaling these values should be constant; they are not, but they have indeed a plateau-like behavior for $2 \mathrm{GeV}<\mu<4 \mathrm{GeV}$ with less than $5 \%$ variation $\left(10 \%\right.$ for $Z_{S}^{\mathrm{RGI}}$ ).

In Tables 1 , 3 we quote the RGI values at $a \mu=1.08$ (the actual values of a $\mu$ closest to 1$)$.

\section{Summary and conclusion}

We have determined the renormalization constants of bilinear quark operators for the chirally improved lattice Dirac operator by non-perturbative methods. These allow us to relate certain lattice observables to those in continuum renormalization schemes. We observe reasonable agreement with 3-loop renormalization group behavior, which improves as the lattice spacing becomes smaller. Finite size effects and problems from Gribov copies were found to be negligible. The Goldstone boson pole in the renormalization factor $Z_{P}$ of the pseudoscalar density could be subtracted leaving behind a well-behaved $Z_{P}^{\mathrm{Sub}}$. The renormalization constants for the vector and the axial vector currents are close to 1 . The ratios $Z_{A} / Z_{V} \approx 1.03$ and $Z_{P}^{\mathrm{Sub}} / Z_{S} \approx 0.95$ for the smallest lattice spacing indicate a very good approximation of chiral symmetry.

Acknowledgments: The quark propagators have been determined on the Hitachi SR8000 of the Leibniz Rechenzentrum Munich. We wish to thank Vladimir Braun, Christian Hoelbling, Stefan Schaefer, and Andreas Schäfer for interesting discussions and support. Support by Fonds zur Förderung der Wissenschaftlichen Forschung in Österreich, project FWF P16310-N08, by Österreichische Akademie der Wissenschaften (APART 654), DFG (Forschergruppe "Gitter-Hadronen-Phänomenologie"), and BMBF is gratefully acknowledged. 


\section{A Tables of results}

\begin{tabular}{r|l|l|l|l|l}
$\mu^{2}\left[\mathrm{GeV}^{2}\right]$ & $Z_{S}$ & $Z_{V}$ & $Z_{T}$ & $Z_{A}$ & $Z_{P}^{\text {Sub }}$ \\
\hline 0.017 & $0.7(1)$ & $0.65(8)$ & $1.0(1)$ & $1.1(1)$ & $0.98(2)$ \\
0.291 & $0.878(6)$ & $0.869(4)$ & $1.209(6)$ & $1.180(4)$ & $0.878(9)$ \\
0.702 & $0.84(1)$ & $0.887(2)$ & $1.118(3)$ & $1.071(5)$ & $0.90(2)$ \\
1.251 & $0.862(3)$ & $0.9163(8)$ & $1.074(2)$ & $1.030(2)$ & $0.844(7)$ \\
2.073 & $0.901(2)$ & $0.9449(5)$ & $1.0522(3)$ & $1.0249(4)$ & $0.87(1)$ \\
3.307 & $0.957(1)$ & $0.9618(3)$ & $1.0258(4)$ & $1.0186(5)$ & $0.865(1)$ \\
4.677 & $0.9851(6)$ & $0.9662(1)$ & $1.0087(2)$ & $1.0112(2)$ & $0.8999(8)$ \\
6.048 & $0.9948(3)$ & $0.9702(1)$ & $1.00145(6)$ & $1.00744(7)$ & $0.9183(5)$ \\
8.104 & $0.9972(1)$ & $0.97129(8)$ & $0.99205(6)$ & $0.99930(6)$ & $0.9379(4)$ \\
10.297 & $0.99097(7)$ & $0.97088(3)$ & $0.98483(2)$ & $0.99055(2)$ & $0.9491(4)$ \\
12.216 & $0.98833(5)$ & $0.97282(3)$ & $0.98345(2)$ & $0.98797(2)$ & $0.9570(5)$ \\
15.095 & $0.98289(3)$ & $0.97420(3)$ & $0.98132(2)$ & $0.98385(1)$ & $0.9638(4)$ \\
18.110 & $0.97871(2)$ & $0.97502(2)$ & $0.97990(1)$ & $0.98088(1)$ & $0.9676(4)$ \\
20.578 & $0.98051(2)$ & $0.97767(2)$ & $0.98207(2)$ & $0.98282(1)$ & $0.9712(4)$ \\
25.512 & $0.98491(1)$ & $0.98165(2)$ & $0.98603(1)$ & $0.98698(1)$ & $0.9754(4)$ \\
28.116 & $0.98870(1)$ & $0.98398(2)$ & $0.98893(2)$ & $0.99038(2)$ & $0.9772(4)$ \\
\hline $\mathrm{RGI}$ & $0.840(2)$ & $0.9376(5)$ & $1.0785(3)$ & $1.0171(4)$ & $0.815(9)$ \\
\hline$\overline{M S}$ & $1.1309(9)$ & $0.9586(2)$ & $0.9944(3)$ & $1.0087(4)$ & $1.0281(5)$
\end{tabular}

Table 1: Values for the renormalization constants in the RI' scheme for the $16^{3} \times 32$ lattice at $\beta=7.90$ as resulting from the extrapolation to $m=0$. The RGI value is taken at $\mu^{2}=2.073 \mathrm{GeV}^{2}$, i.e. $a \mu=1.07992$. The $\overline{\mathrm{MS}}(2 \mathrm{GeV})$ value is computed by interpolation. 


\begin{tabular}{r|l|l|l|l|l}
$\mu^{2}\left[\mathrm{GeV}^{2}\right]$ & $Z_{S}$ & $Z_{V}$ & $Z_{T}$ & $Z_{A}$ & $Z_{P}^{\text {Sub }}$ \\
\hline 0.036 & $1.7(1)$ & $0.99(1)$ & $1.61(3)$ & $1.95(5)$ & $1.9(3)$ \\
0.613 & $0.86(2)$ & $0.982(4)$ & $1.301(8)$ & $1.22(1)$ & $1.00(6)$ \\
1.479 & $0.779(6)$ & $0.955(3)$ & $1.124(4)$ & $1.039(4)$ & $0.82(2)$ \\
2.633 & $0.842(2)$ & $0.969(2)$ & $1.072(3)$ & $1.020(3)$ & $0.836(9)$ \\
4.365 & $0.906(1)$ & $0.980(1)$ & $1.045(1)$ & $1.017(1)$ & $0.853(3)$ \\
6.962 & $0.9653(6)$ & $0.9806(4)$ & $1.0209(6)$ & $1.0120(6)$ & $0.902(2)$ \\
9.848 & $0.9873(3)$ & $0.9799(3)$ & $1.0065(4)$ & $1.0060(4)$ & $0.9277(8)$ \\
12.733 & $0.9945(2)$ & $0.9807(3)$ & $1.0004(3)$ & $1.0034(3)$ & $0.9431(3)$ \\
17.062 & $0.9955(2)$ & $0.9802(1)$ & $0.9936(1)$ & $0.9976(1)$ & $0.9576(4)$ \\
21.679 & $0.99084(6)$ & $0.97923(8)$ & $0.98829(7)$ & $0.99153(7)$ & $0.9653(5)$ \\
25.719 & $0.98908(5)$ & $0.98027(6)$ & $0.98728(5)$ & $0.98978(5)$ & $0.9704(5)$ \\
31.780 & $0.98535(3)$ & $0.98090(4)$ & $0.98563(3)$ & $0.98683(3)$ & $0.9747(5)$ \\
38.128 & $0.98235(2)$ & $0.98116(3)$ & $0.98446(2)$ & $0.98464(2)$ & $0.9769(5)$ \\
43.323 & $0.98373(2)$ & $0.98284(3)$ & $0.98589(2)$ & $0.98600(2)$ & $0.9791(5)$ \\
53.712 & $0.98695(3)$ & $0.98538(3)$ & $0.98854(3)$ & $0.98892(3)$ & $0.9818(5)$ \\
59.194 & $0.98961(4)$ & $0.98687(3)$ & $0.99047(3)$ & $0.99125(3)$ & $0.9828(5)$ \\
65.543 & $0.99425(4)$ & $0.98943(3)$ & $0.99383(3)$ & $0.99532(4)$ & $0.9847(5)$ \\
\hline RGI & $0.751(1)$ & $0.975(1)$ & $1.115(1)$ & $1.011(1)$ & $0.708(2)$ \\
\hline$\overline{\text { MS }}$ & $1.039(1)$ & $0.973(1)$ & $1.028(2)$ & $1.012(1)$ & $0.987(4)$
\end{tabular}

Table 2: Values for the renormalization constants in the RI' scheme for the $16^{3} \times 32$ lattice at $\beta=8.35$ as resulting from the extrapolation to $m=0$. The RGI value is taken at $\mu^{2}=4.365 \mathrm{GeV}^{2}$, i.e. $a \mu=1.07992$. The $\overline{\mathrm{MS}}(2 \mathrm{GeV})$ value is computed by interpolation. 


\begin{tabular}{r|l|l|l|l|l}
$\mu^{2}\left[\mathrm{GeV}^{2}\right]$ & $Z_{S}$ & $Z_{V}$ & $Z_{T}$ & $Z_{A}$ & $Z_{P}^{\text {Sub }}$ \\
\hline 0.062 & $1.73(9)$ & $1.08(1)$ & $1.84(3)$ & $2.16(5)$ & $0.64(4)$ \\
0.555 & $0.89(2)$ & $1.078(8)$ & $1.52(1)$ & $1.44(1)$ & $0.838(7)$ \\
1.049 & $0.764(8)$ & $1.001(5)$ & $1.276(7)$ & $1.180(7)$ & $0.755(5)$ \\
2.529 & $0.760(5)$ & $0.968(3)$ & $1.101(3)$ & $1.018(3)$ & $0.744(2)$ \\
3.023 & $0.787(3)$ & $0.972(1)$ & $1.088(1)$ & $1.015(2)$ & $0.746(2)$ \\
3.516 & $0.809(2)$ & $0.982(1)$ & $1.079(1)$ & $1.0165(9)$ & $0.782(1)$ \\
4.503 & $0.842(2)$ & $0.9876(7)$ & $1.065(1)$ & $1.0139(8)$ & $0.797(1)$ \\
5.984 & $0.887(1)$ & $0.9881(7)$ & $1.0496(9)$ & $1.0139(9)$ & $0.834(1)$ \\
7.464 & $0.917(2)$ & $0.9869(7)$ & $1.0366(8)$ & $1.0121(9)$ & $0.8669(8)$ \\
11.905 & $0.9652(3)$ & $0.9851(2)$ & $1.0161(3)$ & $1.0081(3)$ & $0.9090(2)$ \\
14.866 & $0.9780(2)$ & $0.9831(1)$ & $1.0078(2)$ & $1.0043(2)$ & $0.9274(2)$ \\
19.308 & $0.9896(2)$ & $0.98369(8)$ & $1.0020(1)$ & $1.0027(1)$ & $0.9431(4)$ \\
21.775 & $0.9933(2)$ & $0.98363(8)$ & $0.9996(1)$ & $1.0015(1)$ & $0.9506(3)$ \\
29.177 & $0.99479(8)$ & $0.98312(3)$ & $0.99397(5)$ & $0.99705(7)$ & $0.9632(4)$ \\
31.645 & $0.99373(8)$ & $0.98255(3)$ & $0.99217(5)$ & $0.99514(6)$ & $0.9655(4)$ \\
37.073 & $0.99102(5)$ & $0.98225(2)$ & $0.98963(3)$ & $0.99202(4)$ & $0.9699(4)$ \\
43.982 & $0.98976(3)$ & $0.98310(2)$ & $0.98881(2)$ & $0.99064(2)$ & $0.9741(4)$ \\
\hline RGI & $0.713(1)$ & $0.9831(7)$ & $1.1300(9)$ & $1.0081(8)$ & $0.6739(6)$ \\
\hline$\overline{M S}$ & $0.959(2)$ & $0.979(1)$ & $1.049(1)$ & $1.0095(7)$ & $0.915(1)$
\end{tabular}

Table 3: Values for the renormalization constants in the RI' scheme for the $16^{3} \times 32$ lattice at $\beta=8.70$ as resulting from the extrapolation to $m=0$. The RGI value is taken at $\mu^{2}=7.464 \mathrm{GeV}^{2}$, i.e. $a \mu=1.07992$. The $\overline{\mathrm{MS}}(2 \mathrm{GeV})$ value is computed by interpolation. 


\section{References}

[1] P. H. Ginsparg and K. G. Wilson, Phys. Rev. D 25 (1982) 2649.

[2] M. Lüscher, Phys. Lett. B 428 (1998) 342.

[3] T. Blum et al., Phys. Rev. D 66 (2002) 014504; A. Yamaguchi, arXiv:hep-lat/0309135; J. B. Zhang et al. Nucl. Phys. Proc. Suppl. 128 (2004) 240.

[4] R. Narayanan and H. Neuberger, Phys. Lett. B 302 (1993) 62.

[5] R. Narayanan and H. Neuberger, Nucl. Phys. B 443 (1995) 305.

[6] P. Hasenfratz and F. Niedermayer, Nucl. Phys. B 414 (1994) 785.

[7] C. Gattringer, Phys. Rev. D 63 (2001) 114501.

[8] D. B. Kaplan, Phys. Lett. B 288 (1992) 342.

[9] V. Furman and Y. Shamir, Nucl. Phys. B 439 (1995) 54.

[10] C. Gattringer et al., Nucl. Phys. B 677 (2004) 3.

[11] C. Gattringer, I. Hip, and C. B. Lang, Nucl. Phys. B 597 (2001) 451.

[12] V. M. Braun et al., Phys. Rev. D 68 (2003) 054501.

[13] http://physik.uni-graz.at/ cbl/research/data/dci/, 2003.

[14] C. Gattringer et al., Nucl. Phys. B (Proc. Suppl.) 119 (2003) 796.

[15] G. Martinelli, C. Pittori, C. T. Sachrajda, M. Testa, and A. Vladikas, Nucl. Phys. B 445 (1995) 81.

[16] M. L. Paciello, S. Petrarca, B. Taglienti, and A. Vladikas, Phys. Lett. B341 (1994) 187.

[17] L. Giusti, M. L. Paciello, C. Parrinello, S. Petrarca, and B. Taglienti, Int. J. Mod. Phys. A16 (2001) 3487.

[18] L. Giusti, S. Petrarca, B. Taglienti, and N. Tantalo, Phys. Lett. B 541 (2002) 350 . 
[19] M. Göckeler et al., Nucl. Phys. B 544 (1999) 699.

[20] H. Suman and K. Schilling, hep-lat/9306018.

[21] J. E. Mandula and M. Ogilvie, Phys. Lett. B248 (1990) 156.

[22] M. Lüscher and P. Weisz, Commun. Math. Phys. 97 (1985) 59.

[23] C. Gattringer, R. Hoffmann, and S. Schaefer, Phys. Rev. D 65 (2002) 094503.

[24] J.-R. Cudell, A. Le Yaouanc, and C. Pittori, Phys. Lett. B454 (1999) 105; ibid. B516 (2001) 92.

[25] L. Giusti and A. Vladikas, Phys. Lett. B488 (2000) 303.

[26] D. Bećirević, V. Gimenez, V. Lubicz, G. Martinelli, M. Papinutto, and J. Reyes, hep-lat/0401033.

[27] H. Pagels, Phys. Rev. D19 (1979) 3080.

[28] E. Franco and V. Lubicz, Nucl. Phys. B 531 (1998) 641.

[29] K. G. Chetyrkin and A. Rétey Nucl. Phys. B 583 (2000) 3.

[30] J. A. Gracey, Nucl. Phys. B 662 (2003) 247.

[31] S. Capitani, M. Lüscher, R. Sommer, and H. Wittig, Nucl. Phys. B 544 (1999) 669.

[32] V. Gimenez, L. Giusti, F. Rapuano, and M. Talevi, Nucl. Phys. B 531 (1998) 429. 